\title{
WELL-POSEDNESS OF SECOND ORDER EVOLUTION EQUATION ON DISCRETE TIME
}

\author{
AIRTON CASTRO, CLAUDIO CUEVAS AND CARLOS LIZAMA
}

\begin{abstract}
We characterize the well-posedness for second order discrete evolution equations in $U M D$ spaces by means of Fourier multipliers and $R$-boundedness properties of the resolvent operator which defines the equation. Applications to semilinear problems are given.
\end{abstract}

\section{INTRODUCTION}

Let $X$ be a Banach space and let $A$ be a bounded linear operator. Our main objective of this paper is to characterize the well-posedness in weighted spaces $l_{p}^{r}\left(\mathbb{Z}_{+} ; X\right):=\left\{\left(x_{n}\right)\right.$ : $\left.\left(r^{-n} x_{n}\right) \in l_{p}\left(\mathbb{Z}_{+} ; X\right)\right\}(r>0)$ for the following discrete second order evolution equation:

$$
\Delta^{2} u_{n}+A u_{n}=f_{n}, \quad n \in \mathbb{Z}_{+},
$$

with zero initial conditions and $f \in l_{p}^{r}\left(\mathbb{Z}_{+} ; X\right)$.

Beside its theoretical interest, the study of abstract discrete evolution equation together with well-posedness has great importance and there is much interest in developing the qualitative theory for such equations. Indeed, this has been strongly motivated by their natural and widespread applicability in several fields of sciences and technology, see e.g. $[1,25,35]$.

For example S. Blunck considered, in [12, 13], maximal regularity of first order evolution equations on discrete time; see also Portal [37, 38], where he discussed discrete analytic semigroup and maximal regularity on discrete time scales, respectively. In [29], maximal regularity on discrete Hölder spaces for finite difference operators subject to Dirichlet boundary conditions in one and two dimensions is proved. Furthermore, the authors investigated maximal regularity in discrete Hölder spaces for the Crank- Nicolson scheme. In [7], well-posedness of difference schemes for abstract elliptic problems in $L^{p}$ spaces was studied. In [26], maximal regularity for linear parabolic difference equations is treated while in $[21,22]$ the authors has made a perturbation theory for semilinear evolution equations on discrete time for first and second order using discrete maximal regularity; see also the recent paper by Kalton and Portal [30], where they discussed maximal regularity of power-bounded operators and relate the discrete to the continuous time problem for

2000 Mathematics Subject Classification. 39A12, 39A11, $47 \mathrm{D} 06$.

Key words and phrases. Well-posedness; Discrete maximal regularity; Second order evolution equations; $R$-boundedness; UMD spaces; Fourier Multipliers; Discrete time.

Corresponding author: cch@dmat.ufpe.br (Claudio Cuevas).

The second author is partially supported by CNPQ/Brazil.

The third author is partially financed by Laboratorio de Análisis Estocástico, Proyecto Anillo PBCT ACT-13. 
analytic semigroups. Recently, discrete maximal regularity for functional difference equations with infinite delay was considered in [20]. There, applications to Volterra difference equations with infinite delay are also shown.

A motivation for our studies in this paper stems in the recent article by Arendt and $\mathrm{Bu}$ [4] and Cuevas and Lizama [19]. In the first one the authors consider the operator-valued Marcinkiewich multiplier theorem and maximal regularity, and the second one the authors shown a characterization for the maximal regularity for a second order difference equation by $R$-boundedness properties of the resolvent operator which defines the equation.

We remark that the concept of $R$-boundedness was implicity introduced by Bourgain in [14] and later on also by Zimmermann [41]. Explicitly it is due to Berkson and Gillespie [11] and Clément et al. [17]. It plays a fundamental role in recent works by Clément-Da Prato [16], Weis [39, 40], Arendt-Bu [4, 5], Keyantuo-Lizama [31, 32, 33] and Ashyralyev et al. $[7,8]$.

One of the most important tools to prove well-posedness are Fourier multiplier theorems. They plays a key role in the analysis of elliptic and parabolic problems. In recent years it has become apparent that one need not only the classical theorems but also vector-valued extensions with operator-valued multiplier functions or symbols. In particular, in [19] the authors used a vector-valued Fourier multiplier theorem due to Blunck (see [12], Theorem 1.3), to study maximal regularity of second order difference equations. These approach will be also our method in this paper.

For an overview of the organization of the work. The second section provides the definitions and preliminary results to be used. In particular to facilitate a comprehensive understanding to the reader we have supplied several basic $R$-boundedness properties which are a natural tool in our setting. We also introduce the concept of $l_{p}-$ multiplier by using Z-transform. In the third section, we show how $R$-boundedness and $l_{p}$-multipliers can be used to obtain a characterization about well-posedness for equation (1.1) in $U M D$ Banach spaces (see Theorem 3.4). By using our characterization and the Implicit Function Theorem, we obtain as an application the existence of solutions to the discrete semilinear evolution equation

$$
\Delta_{r}^{2} x-r^{2}(I-T) x=G(x)+\rho f,
$$

where $G$ is a Frechét differentiable function satisfying suitable assumptions and $\rho>0$ is a small parameter (see Theorem 3.7 and Corollary 3.8).

\section{Preliminaries}

Let $X$ be a Banach space. Let $\mathbb{Z}_{+}$denote the set of positive integer numbers, the $\Delta_{r}$ denotes the $r$-difference operator of the first order, i.e. for each $x: \mathbb{Z}_{+} \rightarrow X$, and $n \in \mathbb{Z}_{+}$, $\Delta_{r} x_{n}=x_{n+1}-r x_{n}, r \in \mathbb{R}_{+}$. In case $r=1$ we denote $\Delta \equiv \Delta_{1}$. Moreover, we denote $\Delta_{r}^{2} x_{n}=\Delta_{r}\left(\Delta_{r} x_{n}\right)$. If we set $\left(\tau_{r} x\right)(n):=r^{-n} x(n)$, then is easy to check that the following identity holds:

$$
\Delta_{r}^{2}=r^{2} \tau_{r^{-1}} \circ \Delta^{2} \circ \tau_{r} .
$$

It shows that well-posedness of equation $(1.1)$ in the weighted spaces $l_{p}^{r}\left(\mathbb{Z}_{+} ; X\right)$ is equivalent to the study of the discrete time evolution equation

$$
\Delta_{r}^{2} x_{n}-r^{2}(I-T) x_{n}=f_{n}, \text { for all } n \in \mathbb{Z}_{+}, \quad x_{0}=0, \quad x_{1}=0 .
$$


in the usual spaces $l_{p}\left(\mathbb{Z}_{+} ; X\right)$, where $T:=I-A \in \mathcal{B}(X)$ and $f: \mathbb{Z}_{+} \rightarrow X$.

Denote $\mathcal{C}(0)=I$, the identity operator on $X$, and define

$$
\mathcal{C}(n)=\sum_{k=0}^{[n / 2]}\left(\begin{array}{c}
n \\
2 k
\end{array}\right)(I-T)^{k}, \quad \text { for } n=1,2, \ldots
$$

We define also $\mathcal{S}(0)=0$,

$$
\mathcal{S}(n)=\sum_{k=0}^{[(n-1) / 2]}\left(\begin{array}{c}
n \\
2 k+1
\end{array}\right)(I-T)^{k},
$$

for $n=1,2, \ldots$ The sequences of linear and bounded operators $\mathcal{C}(n)$ and $\mathcal{S}(n)$ were introduced in [19] to represent the solution of (2.2) in the border case $r=1$. The following result generalize [19, Proposition 2.2].

Proposition 2.1. Let $T \in \mathcal{B}(X)$ be given, then the (unique) solution of equation (2.2) is given by

$$
x_{m+1}=\left(r^{\bullet-1} \mathcal{S} * f\right)_{m} .
$$

Moreover,

$$
\Delta_{r} x_{m+1}=\left(r^{\bullet} \mathcal{C} * f\right)_{m} .
$$

Proof. Let $x_{n}$ be the solution of equation (2.2) and define $v_{n}:=\left[x_{n}, \Delta_{r} x_{n}\right], F_{n}:=\left[0, f_{n}\right]$ and the operator $R_{T, r} \in \mathcal{B}(X \times X)$ by

$$
R_{T, r}[x, y]=\left[r x+y, r^{2}(x-T x)+r y\right] .
$$

Then, we can infer that the equation (2.2) is equivalent to:

$$
v_{n+1}-R_{T, r} v_{n}=F_{n}, \quad v_{0}=[0,0],
$$

which has the solution

$$
v_{m+1}=\sum_{n=0}^{m} R_{T, r}^{n} F_{m-n}
$$

Denote

$$
R_{T, r}=\left[\begin{array}{cc}
r I & I \\
r^{2}(I-T) & r I
\end{array}\right] .
$$

Then a calculation shows us that

$$
R_{T, r}^{n}=r^{n}\left[\begin{array}{cc}
\mathcal{C}(n) & \frac{1}{r} \mathcal{S}(n) \\
r(I-T) \mathcal{S}(n) & \mathcal{C}(n)
\end{array}\right] .
$$

The result is now a consequence of formula (2.8). The uniqueness follows from induction and then the proof is finished. 
Definition 2.2. Let $1<p<+\infty$. One says that equation (2.2) has discrete maximal regularity if $\mathcal{K}^{r} f:=(I-T) r^{\bullet+1} \mathcal{S} * f$ defines a linear bounded operator $\mathcal{K}^{r} \in \mathcal{B}\left(l_{p}\left(\mathbb{Z}_{+}, X\right)\right)$.

Note that, in particular, the definition implies that for all $\left(f_{n}\right) \in l_{p}\left(\mathbb{Z}_{+}, X\right)$ we have $\left(\Delta_{r}^{2} x_{n}\right) \in l_{p}\left(\mathbb{Z}_{+}, X\right)$ in equation $(2.2)$. This is the main property that allows the use of maximal regularity in the treatment of non-linear problems.

We introduce the means

$$
\left\|\left(x_{1}, \ldots, x_{n}\right)\right\|_{R}:=\frac{1}{2^{n}} \sum_{\epsilon_{j} \in\{-1,1\}^{n}}\left\|\sum_{j=1}^{n} \epsilon_{j} x_{j}\right\|,
$$

for $x_{1}, \ldots, x_{n} \in X$.

Let $X$ and $Y$ be Banach spaces, $\mathcal{B}(X, Y)$ be the space of bounded linear operators from $X$ to $Y$.

Definition 2.3. A subset $\mathcal{T}$ of $\mathcal{B}(X, Y)$ is called $R$-bounded if there exists a constant $c \geq 0$ such that

$$
\left\|\left(T_{1} x_{1}, \ldots, T_{n} x_{n}\right)\right\|_{R} \leq c\left\|\left(x_{1}, \ldots, x_{n}\right)\right\|_{R},
$$

for all $T_{1}, \ldots, T_{n} \in \mathcal{T}, x_{1}, \ldots, x_{n} \in X, n \in \mathbb{N}$. The least $c$ such that (2.9) is satisfied is called the $R$-bound of $\mathcal{T}$ and is denoted $R(\mathcal{T})$.

The notion of $R$-boundedness has proved to be a significant tool in the study of abstract multiplier operators. An equivalent definition using the Rademacher functions can be found in [23]. We note that $R$-boundedness clearly implies boundedness. If $X=Y$, the notion of $R$-boundedness is strictly stronger than boundedness unless the underlying space is isomorphic to a Hilbert space, see [4, Proposition 1.17]. Some useful criteria for $R$-boundedness are provided in [4, 23] and [28].

Remark 2.4. a) Any finite family $\mathcal{T} \subset \mathcal{B}(X, Y)$ is $R$-bounded.

b) If $\mathcal{T} \subset \mathcal{B}(X, Y)$ is $R$-bounded then it is uniformly bounded, with $\sup \{\|T\|: T \in$ $\mathcal{T}\} \leq R_{p}(\mathcal{T})$.

c) The definition of $R$-boundedness is independent of $p \in[1, \infty)$.

d) When $X$ and $Y$ are Hilbert spaces, $\mathcal{T} \subset \mathcal{B}(X, Y)$ is $R$-bounded if and only if $\mathcal{T}$ is uniformly bounded.

e) Let $\mathcal{S}, \mathcal{T} \subset \mathcal{B}(X, Y)$ be $R$-bounded sets, then $\mathcal{S} \pm \mathcal{T}:=\{S \pm T: S \in \mathcal{S}, T \in \mathcal{T}\}$ are $R$ - bounded.

f) Let $\mathcal{T} \subset \mathcal{B}(X, Y)$ and $\mathcal{S} \subset \mathcal{B}(Y, Z)$ be $R$-bounded sets, then $\mathcal{S} \cdot \mathcal{T}:=\{S \cdot T: S \in$ $\mathcal{S}, T \in \mathcal{T}\} \subset \mathcal{B}(X, Z)$ is $R$-bounded and $R(\mathcal{S} \cdot \mathcal{T}) \leq R(\mathcal{S}) \cdot R(\mathcal{T})$.

g) Also, each subset $M \subset \mathcal{B}(X)$ of the form $M=\{\lambda I: \lambda \in \Omega\}$ is $R$ - bounded, whenever $\Omega \subset \mathbb{C}$ is bounded (see Example 3.2 in $[12]$ ).

Definition 2.5. A Banach space $X$ is said to have the unconditional martingale difference property (UMD) if for each $p \in(1, \infty)$ there is a constant $C_{p}>0$ such that for any martingale $\left(f_{n}\right)_{n \geq 0} \subset L^{p}(\Omega, \Sigma, \mu ; X)$ and any choice of signs $\left(\xi_{n}\right)_{n \geq 0} \subset\{-1,1\}$ and any $N \in \mathbb{Z}_{+}$the following estimate holds

$$
\left\|f_{0}+\sum_{n=1}^{N} \xi_{n}\left(f_{n}-f_{n-1}\right)\right\|_{L^{p}(\Omega, \Sigma, \mu ; X)} \leq C_{p}\left\|f_{N}\right\|_{L^{p}(\Omega, \Sigma, \mu ; X)} .
$$


Remark 2.6. A Banach space $X$ is said to be $\mathcal{H} \mathcal{T}$, if the Hilbert transform is bounded on $L^{p}(\mathbb{R}, X)$ for some (and then all) $p \in(1, \infty)$. Here, the Hilbert transform $H$ of a function $f \in \mathcal{S}(\mathbb{R}, X)$, the Schwartz space of rapidly decreasing $X$-valued functions, is defined by

$$
H f:=\frac{1}{\pi} P V\left(\frac{1}{t}\right) * f .
$$

It is a well known theorem that the set of Banach spaces of class $\mathcal{H} \mathcal{T}$ coincides with the class of $U M D$ spaces. This has been shown by Bourgain [14] and Burkholder [15]. For more information and details on the Hilbert transform and the $U M D$ Banach spaces we refer to Amann's book [3, Section III.4.3-III.4.5]. The $U M D$ spaces include Hilbert spaces, Sobolev spaces $W_{p}^{s}(\Omega), 1<p<\infty$ (see [4]), Lebesgue spaces $L^{p}(\Omega, \mu), L^{p}(\Omega, \mu ; X)$, $1<p<\infty$, where $X$ is a $U M D$ space and the Schatten-von Neumann classes $C_{p}(H), 1<$ $p<\infty$, of operators on Hilbert spaces. On the other hand, we can observe that:

a) Every closed subspace of a $U M D$ space is a $U M D$ space.

b) Every $U M D$ space is reflexive.

c) A Banach space $X$ is $U M D$ if and only if its dual $X^{*}$ is $U M D$.

We recall now the following operator-valued multiplier theorem on $\mathbb{T}:=\{z \in \mathbb{C}:|z|=$ 1) due to Blunck, see [12, Theorem 1.3].

Theorem 2.7. Let $p \in(1, \infty)$ and $X$ be a $U M D$ space. Let $M:(-\pi, \pi) \backslash\{0\} \rightarrow \mathcal{B}(X)$ be a differentiable function such that the set

$$
\left\{M(t),\left(e^{i t}-1\right)\left(e^{i t}+1\right) M^{\prime}(t): t \in(-\pi, \pi) \backslash\{0\}\right\}
$$

is $R$-bounded. Then there is an operator $T_{M} \in \mathcal{B}\left(l_{p}(\mathbb{Z} ; X)\right)$ such that

$$
\mathcal{F}\left(T_{M} f\right)\left(e^{i t}\right)=M(t) \mathcal{F} f\left(e^{i t}\right), \quad t \in(-\pi, \pi) \backslash\{0\}, \mathcal{F} f \in L_{\infty}(\mathbb{T} ; X)
$$

of compact support.

Definition 2.8. An operator $S \in \mathcal{B}(X)$ is called analytic if the set $\left\{n(S-I) S^{n}: n \in \mathbb{N}\right\}$ is bounded.

This notion is a discrete analogue of the property " $\{t A \exp (t A): t>0\}$ is bounded", which characterizes the analyticity of the bounded semigroup $(\exp (t A))_{t \geq 0}$. For recent and related results on analytic operators we refer the reader to [24]. In what follows, we denote $\mathbb{D}(z, a)=\{w \in \mathbb{C}:|w-z|<a\}$.

Proposition 2.9. Let $T \in \mathcal{B}(X)$ be an analytic operator. Then $\sigma\left(r^{2}(I-T)\right) \subseteq \mathbb{D}\left(r^{2}, r^{2}\right) \cup$ $\{0\}$. In particular $(z-r)^{2} \in \rho\left(r^{2}(I-T)\right)$ whenever $|z|=\alpha r, \alpha=1+\sqrt{2}, z \neq \alpha r$.

Proof. Let $z \notin \mathbb{D}\left(r^{2}, r^{2}\right) \cup\{0\}$, then $\frac{z}{r^{2}} \notin \mathbb{D}(1,1) \cup\{0\}$. By Lemma 2.10 Ref. [19], we get $\frac{z}{r^{2}} \in \rho(I-T)$, that is, $z \in \rho\left(r^{2}(I-T)\right)$. Hence $\sigma\left(r^{2}(I-T)\right) \subseteq \mathbb{D}\left(r^{2}, r^{2}\right) \cup\{0\}$. For the last assertion, we note that $\left|(z-r)^{2}-r^{2}\right|=|z||z-2 r| \geq \alpha(\alpha-2) r^{2}=(\sqrt{2}+1)(\sqrt{2}-1) r^{2}=r^{2}$.

For the rest of the article, we will always assume that

$$
\alpha=1+\sqrt{2}, r \geq r_{0}, 1 /(1+\sqrt{2})<r_{0}<1 .
$$


We recall that the Z-transform on $\mathbb{T}_{r}^{\alpha}:=\{z \in \mathbb{C}:|z|=\alpha r\}$ is defined as

$$
\mathcal{F} f(z)=\hat{f}(z)=\sum_{j=0}^{\infty} z^{-j} f(j), \quad z \in \mathbb{T}_{r}^{\alpha} .
$$

It follows from Hölder inequality that the Z-transform, $\hat{f}(z)$, is well defined for all $f \in$ $l_{p}\left(\mathbb{Z}_{+} ; X\right)$. Moreover, we can relate the Fourier transform of $f$ on $\mathbb{T}_{r}^{\alpha}$ with the Fourier transform of $(\alpha r)^{-\bullet} f$ on $\mathbb{T}_{1}^{1}$ by the formula

$$
\mathcal{F}[f]\left(\alpha r e^{i t}\right)=\mathcal{F}\left[(\alpha r)^{-\bullet} f\right]\left(e^{i t}\right) .
$$

This observation will be very useful in the third section in connection with Theorem 2.7. The preceding proposition enables us to prove the following properties on the Fourier transform of the solution of $(2.2)$.

Proposition 2.10. Let $T \in \mathcal{B}(X)$ be an analytic operator. Then

$$
\mathcal{F}\left[r^{\bullet-1} \mathcal{S}\right](z)=z R\left((z-r)^{2}, r^{2}(I-T)\right), \quad z \in \mathbb{T}_{r}^{\alpha} \backslash\{\alpha r\},
$$

$$
\mathcal{F}\left[r^{\bullet} \mathcal{C}\right](z)=z(z-r) R\left((z-r)^{2}, r^{2}(I-T)\right), \quad z \in \mathbb{T}_{r}^{\alpha} \backslash\{\alpha r\} .
$$

Proof. Given $x \in X$, we define

$$
f_{n}=\left\{\begin{array}{cc}
x, & \text { for } n=0 \\
0, & \text { for } n \neq 0 .
\end{array}\right.
$$

A direct calculation shows that $\hat{f}(z)=x$. We consider the following evolution problem

$$
\Delta_{r}^{2} x_{n}-r^{2}(I-T) x_{n}=f_{n} \text { for all } n \in \mathbb{Z}_{+}, \quad x_{0}=x_{1}=0 .
$$

By Proposition 2.1 the (unique) solution is given by $x_{n+1}=\left(r^{\bullet-1} \mathcal{S} * f\right)(n)$. Then $z \hat{x}(z)=$ $\mathcal{F}\left[r^{\bullet-1} \mathcal{S}\right](z) x, z \in \mathbb{T}_{r}^{\alpha}$. On the other hand, we note that a direct calculation gives $\widehat{\Delta_{r} x}(z)=$ $(z-r) \hat{x}(z)$, for $z \in \mathbb{T}_{r}^{\alpha}$. Hence, applying Z-transform in (2.13) and then multiplying the result by $z$, we obtain $z x=\left((z-r)^{2}-r^{2}(I-T)\right) \mathcal{F}\left[r^{\bullet-1} \mathcal{S}\right](z) x, z \in \mathbb{T}_{r}^{\alpha}$, obtaining the first assertion. To prove the second one, we note that by Proposition $2.1 \Delta_{r} x_{n+1}=\left(r^{\bullet} \mathcal{C} * f\right)(n)$ and then $z(z-r) \hat{x}(z)=\mathcal{F}\left[r^{\bullet} \mathcal{C}\right](z) x, z \in \mathbb{T}_{r}^{\alpha}$. Therefore, applying Z-transform in (2.13) and then multiplying the result by $z(z-r)$, we get the second assertion and the proof is finished.

Next, we consider the following sequence spaces; for $r \geq r_{0}$

$$
\begin{gathered}
l_{p, r}^{1}\left(\mathbb{Z}_{+} ; X\right):=\left\{y=\left(y_{n}\right) / y_{0}=0,\left(\Delta_{r} y_{n}\right) \in l_{p}\left(\mathbb{Z}_{+} ; X\right)\right\}, \\
l_{p, r}^{2}\left(\mathbb{Z}_{+} ; X\right):=\left\{y=\left(y_{n}\right) / y_{0}=y_{1}=0,\left(\Delta_{r}^{2} y_{n}\right) \in l_{p}\left(\mathbb{Z}_{+} ; X\right)\right\}, \\
l_{p, I-T}\left(\mathbb{Z}_{+} ; X\right):=\left\{y=\left(y_{n}\right) /\left((I-T) y_{n}\right) \in l_{p}\left(\mathbb{Z}_{+} ; X\right)\right\} .
\end{gathered}
$$

Proposition 2.11. Assume that (2.10) is fulfilled. For each $y \in l_{p, r}^{i}\left(\mathbb{Z}_{+} ; X\right), i=1,2$, the Z-transform of $y$ is well defined in $\mathbb{T}_{r}^{\alpha}$. 
Proof. For $y \in l_{p, r}^{1}\left(\mathbb{Z}_{+} ; X\right)$, we have

$$
\begin{aligned}
\|\hat{y}(z)\|_{X} & =\sum_{j=0}^{\infty}|z|^{-j}\|y(j)\|_{X} \\
& =\sum_{j=0}^{\infty}\left(\frac{1}{\alpha r}\right)^{j}\left\|\sum_{i=0}^{j-1} r^{i} \Delta_{r} y_{j-1-i}\right\|_{X} \\
& \leq \sum_{j=0}^{\infty}\left(\frac{1}{\alpha r}\right)^{j} \sum_{i=0}^{j-1} r^{i}\left\|\Delta_{r} y_{j-1-i}\right\|_{X} \\
& \leq C(\alpha, r)\left\|\Delta_{r} y_{\bullet}\right\|_{\infty},
\end{aligned}
$$

where $C(\alpha, r)$ is a constant depending on $\alpha$ and $r$. On the other hand, for $y \in l_{p, r}^{2}\left(\mathbb{Z}_{+} ; X\right)$, we can infer that

$$
\begin{aligned}
\|\hat{y}(z)\|_{X} & \leq \sum_{j=0}^{\infty}\left(\frac{1}{\alpha r}\right)^{j} \sum_{i=0}^{j-1} r^{i} \sum_{k=0}^{j-i-2} r^{k}\left\|\Delta_{r}^{2} y_{j-i-2-k}\right\|_{X} \\
& \leq \sum_{j=0}^{\infty}\left(\frac{1}{\alpha r}\right)^{j} \sum_{i=0}^{j-1} r^{i} \sum_{k=0}^{j-i-2} r^{k}\left\|\Delta_{r}^{2} y_{\bullet}\right\|_{\infty} \\
& \leq C(\alpha, r)\left\|\Delta_{r}^{2} y_{\bullet}\right\|_{\infty},
\end{aligned}
$$

and the proof is finished.

In the following definition we denote $l_{p, r}^{0}\left(\mathbb{Z}_{+} ; X\right):=l_{p}\left(\mathbb{Z}_{+} ; X\right)$.

Definition 2.12. Assume that (2.10) is fulfilled. We say that $\{Q(z)\}_{z \in \mathbb{T}_{r}^{\alpha}}$ is a $l_{p}$ $l_{p, r}^{i}-$ multiplier, $i=0,1,2$, if for each $f=\left(f_{n}\right) \in l_{p}\left(\mathbb{Z}_{+} ; X\right)$ there is a sequence $y=\left(y_{n}\right) \in$ $l_{p, r}^{i}\left(\mathbb{Z}_{+} ; X\right)$ such that $\hat{y}(z)=Q(z) \hat{f}(z), z \in \mathbb{T}_{r}^{\alpha}$.

We finish this section with the following proposition.

Proposition 2.13. Assume that (2.10) is fulfilled. The following assertions are equivalent.

(i) $\{Q(z)\}_{z \in \mathbb{T}_{r}^{\alpha}}$ is a $l_{p}-l_{p, r}^{i}-$ multiplier, $i=1,2$.

(ii) $\left\{(z-r)^{i} Q(z)\right\}_{z \in \mathbb{T}_{r}^{\alpha}}$ is a $l_{p}-l_{p}$-multiplier, $i=1,2$.

Proof. Initially we consider the case $i=1$. To prove $(i) \Rightarrow(i i)$, for $f=\left(f_{n}\right) \in l_{p}\left(\mathbb{Z}_{+} ; X\right)$ there is a sequence $y=\left(y_{n}\right) \in l_{p, r}^{1}\left(\mathbb{Z}_{+} ; X\right)$ such that $\hat{y}(z)=Q(z) \hat{f}(z), z \in \mathbb{T}_{r}^{\alpha}$. Putting $x_{0}=0$, and $x_{n}=\Delta_{r} y_{n}, n \geq 1$, we have $x=\left(x_{n}\right) \in l_{p}\left(\mathbb{Z}_{+} ; X\right)$ and $\hat{x}(z)=\widehat{\Delta_{r} y}(z)=$ $(z-r) Q(z) \hat{f}(z)$. To prove $(i i) \Rightarrow(i)$, for $f=\left(f_{n}\right) \in l_{p}\left(\mathbb{Z}_{+} ; X\right)$ there is a sequence $y=\left(y_{n}\right) \in l_{p}\left(\mathbb{Z}_{+} ; X\right)$ such that $\hat{y}(z)=(z-r) Q(z) \hat{f}(z)$. Let $x=\left(x_{n}\right) \in l_{p, r}^{1}\left(\mathbb{Z}_{+} ; X\right)$ be a 
sequence such that

$$
\left\{\begin{array}{l}
\Delta_{r} x_{n}=y_{n} \\
x_{0}=0
\end{array}\right.
$$

Indeed, we observe that $x_{n}=\sum_{i=0}^{n-1} r^{i} y_{n-1-i}$. Therefore,

$$
(z-r) \hat{x}(z)=\widehat{\Delta_{r} x}(z)=(z-r) Q(z) \hat{f}(z) .
$$

Hence, $Q(z)$ is a $l_{p}-l_{p, r}^{1}-$ multiplier. Finally, we treat the case $i=2$. To prove $(i) \Rightarrow(i i)$, for $f=\left(f_{n}\right) \in l_{p}\left(\mathbb{Z}_{+} ; X\right)$ there is a sequence $y=\left(y_{n}\right) \in l_{p, r}^{2}\left(\mathbb{Z}_{+} ; X\right)$ such that $\hat{y}(z)=$ $Q(z) \hat{f}(z), z \in \mathbb{T}_{r}^{\alpha}$. Putting $x_{n}=\Delta_{r}^{2} y_{n} \in l_{p}\left(\mathbb{Z}_{+} ; X\right)$, we get

$$
\hat{x}(z)=(z-r) \widehat{\Delta_{r} y}(z)-z\left(y_{1}-r y_{0}\right)=(z-r)^{2} Q(z) \hat{f}(z) .
$$

Hence $(z-r)^{2} Q(z)$ is a $l_{p}-l_{p}$-multiplier. To prove $(i i) \Rightarrow(i)$, for $f=\left(f_{n}\right) \in l_{p}\left(\mathbb{Z}_{+} ; X\right)$ there is a sequence $y=\left(y_{n}\right) \in l_{p}\left(\mathbb{Z}_{+} ; X\right)$ such that $\hat{y}(z)=(z-r)^{2} Q(z) \hat{f}(z), z \in \mathbb{T}_{r}^{\alpha}$. Let $x=\left(x_{n}\right) \in l_{p, r}^{2}\left(\mathbb{Z}_{+} ; X\right)$ be a sequence such that

$$
\left\{\begin{array}{l}
\Delta_{r}^{2} x_{n}=y_{n} \\
x_{0}=x_{1}=0
\end{array}\right.
$$

Indeed, we observe that $x_{n}=\sum_{i=0}^{n-1} \sum_{j=0}^{n-i-2} r^{i+j} y_{n-2-i-j}$. Therefore,

$$
(z-r)^{2} \hat{x}(z)=\widehat{\Delta_{r}^{2} x}(z)=(z-r)^{2} Q(z) \hat{f}(z) .
$$

Hence, $Q(z)$ is a $l_{p}-l_{p, r}^{2}-$ multiplier. This completes the proof of the proposition.

\section{A Characterization of Maximal Regularity}

Having presented in the previous sections preliminary material on $R$-boundedness and Fourier multipliers, we will now show how these tools can be used to handle well-posedness of equation (2.2). The following is a natural extension of the concept of well-posedness to the continuous to the discrete case.

Definition 3.1. One says that problem (2.2) is well-posed if for each $f=\left(f_{n}\right) \in l_{p}\left(\mathbb{Z}_{+} ; X\right)$ there is an unique solution $x=\left(x_{n}\right) \in l_{p, r}^{2}\left(\mathbb{Z}_{+} ; X\right) \cap l_{p, I-T}\left(\mathbb{Z}_{+} ; X\right)$ of equation (2.2).

We observe that the space $l_{p, r}^{2}\left(\mathbb{Z}_{+} ; X\right) \cap l_{p, I-T}\left(\mathbb{Z}_{+} ; X\right)$ becomes a Banach space under the norm

$$
\||| x \mid\|:=\left\|\Delta_{r}^{2} x\right\|_{p}+\left\|r^{2}(I-T) x\right\|_{p} .
$$

The following result characterizes well-posedness of (2.2) in terms of $l_{p}$-multipliers.

Proposition 3.2. Let $X$ be a Banach space and let $T \in \mathcal{B}(X)$ be an analytic operator. Assume that (2.10) is fulfilled. Then, the following assertions are equivalent.

(i) Problem (2.2) is well-posed.

(ii) $\left\{M(z):=(z-r)^{2}\left((z-r)^{2}-r^{2}(I-T)\right)^{-1}: z \in \mathbb{T}_{r}^{\alpha}, z \neq \alpha r\right\}$ is a $l_{p}-l_{p}-$ multiplier. 
Proof. $\quad(i) \Rightarrow(i i)$, applying Z-transform to equation (2.2), we get

$$
(z-r)^{2} \hat{x}(z)-r^{2}(I-T) \hat{x}(z)=\hat{f}(z), \quad z \in \mathbb{T}_{r}^{\alpha} .
$$

By Proposition 2.9, we have $\hat{x}(z)=R\left((z-r)^{2}, r^{2}(I-T)\right) \hat{f}(z), z \in \mathbb{T}_{r}^{\alpha}, z \neq \alpha r$; consequently $R\left((z-r)^{2}, r^{2}(I-T)\right)$ is a $l_{p}-l_{p, r}^{2}-$ multiplier. By Proposition 2.13, we conclude the proof of $(i i)$.

$(i i) \Rightarrow(i)$, by Proposition 2.13, $R\left((z-r)^{2}, r^{2}(I-T)\right)$ is a $l_{p}-l_{p, r}^{2}-$ multiplier. Hence, for all $f=\left(f_{n}\right) \in l_{p}\left(\mathbb{Z}_{+} ; X\right)$ there is a sequence $x=\left(x_{n}\right) \in l_{p, r}^{2}\left(\mathbb{Z}_{+} ; X\right)$ so that $(z-r)^{2} \hat{x}(z)-$ $r^{2}(I-T) \hat{x}(z)=\hat{f}(z)$. By uniqueness of the Z-transform, we conclude that $x=\left(x_{n}\right)$ is solution of equation (2.2). In particular, $r^{2}(I-T) x_{n}=\Delta_{r}^{2} x_{n}-f_{n}$ belongs to $l_{p}\left(\mathbb{Z}_{+} ; X\right)$. To prove the uniqueness of the solution, we observe that by application of the Z-transform to equation $(2.2)$ with $f_{n} \equiv 0$, we get $\hat{x}(z)=0$, and so $x \equiv 0$.

Proposition 3.3. Let $X$ be a Banach space and let $T \in \mathcal{B}(X)$ be an analytic operator; assume that (2.10) is fulfilled and suppose that problem (2.2) is well-posed. Then $(z-r)^{2} \in$ $\rho\left(r^{2}(I-T)\right)$ whenever $|z|=\alpha r, z \neq \alpha r$ and the set $\{M(z):|z|=\alpha r, z \neq \alpha r\}$ is $R$ bounded.

Proof. By Proposition 2.9 the first assertion follows. On the other hand, by Proposition 3.2, for all $f=\left(f_{n}\right) \in l_{p}\left(\mathbb{Z}_{+} ; X\right)$, there is a sequence $\mathcal{L}_{M} f \in l_{p}\left(\mathbb{Z}_{+} ; X\right)$ such that $\widehat{\mathcal{L}_{M} f}(z)=M(z) \hat{f}(z), z \in \mathbb{T}_{r}^{\alpha}, z \neq \alpha r$. By the uniqueness of Z-transform the operator $\mathcal{L}_{M}: l_{p}\left(\mathbb{Z}_{+} ; X\right) \rightarrow l_{p}\left(\mathbb{Z}_{+} ; X\right)$ is well defined. It follows from the Closed Graph Theorem that $\mathcal{L}_{M}$ is in $\mathcal{B}\left(l_{p}\left(\mathbb{Z}_{+} ; X\right)\right)$. Now the conclusion is a consequence of [12, Proposition 1.4].

The following is the main result of this paper. It shows that the converse of the above proposition is valid in $U M D$ spaces.

Theorem 3.4. Let $X$ be a $U M D$ space and let $T \in \mathcal{B}(X)$ be an analytic operator; assume that (2.10) is fulfilled. Then, the following assertions are equivalent.

(i) Equation (2.2) is well-posed.

(ii) The set $\{M(z):|z|=\alpha r, z \neq \alpha r\}$ is R-bounded.

(iii) Equation (2.2) has discrete maximal regularity.

Proof. We note that $(i) \Rightarrow(i i)$ is the preceding proposition. To prove $(i i) \Rightarrow(i i i)$, we define $k_{r}: \mathbb{Z} \rightarrow \mathcal{B}(X)$ by

$$
k_{r}(n)= \begin{cases}r^{2}(I-T) r^{n-1} \mathcal{S}(n), & \text { for } n \in \mathbb{Z}_{+}, \\ 0, & \text { otherwise, }\end{cases}
$$

and the corresponding operator $\mathcal{K}^{r}: l_{p}\left(\mathbb{Z}_{+} ; X\right) \rightarrow l_{p}\left(\mathbb{Z}_{+} ; X\right)$ by

$$
\left[\mathcal{K}^{r} f\right](n)=\sum_{j=0}^{n} k_{r}(j) f_{n-j}=\left(k_{r} * f\right)(n), \quad n \in \mathbb{Z}_{+} .
$$

By Proposition 2.9, $(z-r)^{2} \in \rho\left(r^{2}(I-T)\right)$ whenever $|z|=\alpha r, z \neq \alpha r$. Then, by Proposition 2.10 , we have

$$
\hat{k}_{r}(z)=r^{2}(I-T) \mathcal{F}\left[r^{\bullet-1} \mathcal{S}\right](z)=r^{2}(I-T) z R\left((z-r)^{2}, r^{2}(I-T)\right)=z(M(z)-I),
$$


where $z \in \mathbb{T}_{r}^{\alpha}, z \neq \alpha r$.

Define $M(t):=M\left(\alpha r e^{i t}\right), t \in(-\pi, 0) \cup(0, \pi)$. We note that

$$
M^{\prime}(t)=2 i \alpha e^{i t}\left(\alpha e^{i t}-1\right)^{-1}\left[M(t)-M(t)^{2}\right] .
$$

Define $N(t):=\alpha r e^{i t}[M(t)-I]$, then $N(t)$ is $R$-bounded by hypothesis and Remark 2.4(b). A calculation shows that

$$
\begin{aligned}
\left(e^{i t}-1\right)\left(e^{i t}+1\right) N^{\prime}(t) & =i \alpha r e^{i t}\left(e^{i t}-1\right)\left(e^{i t}+1\right)[M(t)-I] \\
& +\frac{2 i \alpha^{2} r e^{2 i t}\left(e^{i t}-1\right)\left(e^{i t}+1\right)}{\alpha e^{i t}-1}\left[M(t)-M(t)^{2}\right] .
\end{aligned}
$$

We observe that $\left|\alpha e^{i t}-1\right| \geq \sqrt{2}$. Hence, the set

$$
\left\{\frac{2 i \alpha^{2} r e^{2 i t}\left(e^{i t}-1\right)\left(e^{i t}+1\right)}{\alpha e^{i t}-1}\right\},
$$

is bounded by $4 \alpha^{2} r \sqrt{2}$. It shows that the set $\left\{\left(e^{i t}-1\right)\left(e^{i t}+1\right) N^{\prime}(t)\right\}$ is $R$-bounded thanks to Remark 2.4 again. We conclude, from Theorem 2.7, that there exists $T_{N} \in \mathcal{B}\left(l_{p}(\mathbb{Z}, X)\right)$ such that $\mathcal{F}\left[T_{N} g\right]\left(e^{i t}\right)=N(t) \mathcal{F}[g]\left(e^{i t}\right)$ for all $g \in l_{p}\left(\mathbb{Z}_{+} ; X\right)$. Let $f \in l_{p}\left(\mathbb{Z}_{+} ; X\right)$ and define $g:=(\alpha r)^{-\bullet} f$. Then $g \in l_{p}\left(\mathbb{Z}_{+} ; X\right)$ because $\alpha r>1$, and we have for all $z \in \mathbb{T}_{r}^{\alpha}$

$$
\begin{aligned}
\mathcal{F}\left[(\alpha r)^{\bullet} T_{N}\left((\alpha r)^{-\bullet} f\right)\right](z) & =\mathcal{F}\left[T_{N}\left((\alpha r)^{-\bullet} f\right)\right]\left(e^{i t}\right) \\
& =N(t) \mathcal{F}\left[(\alpha r)^{-\bullet} f\right]\left(e^{i t}\right) \\
& =N(t) \mathcal{F}[f]\left(\alpha r e^{i t}\right) \\
& =\mathcal{F}\left[k_{r}\right]\left(\alpha r e^{i t}\right) \mathcal{F}[f]\left(\alpha r e^{i t}\right) \\
& =\mathcal{F}\left[k_{r} * f\right]\left(\alpha r e^{i t}\right) \\
& =\mathcal{F}\left[\mathcal{K}^{r} f\right](z) .
\end{aligned}
$$

Then by uniqueness of the Z-transform, we conclude that $(\alpha r)^{-\bullet} \mathcal{K}^{r} f=T_{N}\left((\alpha r)^{-\bullet} f\right)$ and we have

$$
\left\|(\alpha r)^{-\bullet} \mathcal{K}^{r} f\right\|_{p}=\left\|T_{N}\left((\alpha r)^{-\bullet} f\right)\right\|_{p} \leq M\left\|(\alpha r)^{-\bullet} f\right\|_{p} \leq M\|f\|_{p} .
$$

Hence $(\alpha r)^{-\bullet} \mathcal{K}^{r}$ belongs to $\mathcal{B}\left(l_{p}\left(\mathbb{Z}_{+}, X\right)\right)$. It follows from the Closed Graph Theorem that $\mathcal{K}^{r} \in \mathcal{B}\left(l_{p}\left(\mathbb{Z}_{+}, X\right)\right)$ then (iii) follows. To prove $($ iii $) \Rightarrow(i)$, by Proposition 2.1 we known that $x(n)=\left(r^{\bullet-1} \mathcal{S} * f\right)(n-1)$ is the unique solution of equation (2.2). Then $\Delta_{r}^{2} x(n)=r^{2}(I-T)\left(r^{\bullet-1} \mathcal{S} * f\right)(n-1)+f(n)=\left[\mathcal{K}^{r} f\right](n-1)+f(n)$. Hence $x=\left(x_{n}\right) \in$ $l_{p, r}^{2}\left(\mathbb{Z}_{+} ; X\right) \cap l_{p, I-T}\left(\mathbb{Z}_{+} ; X\right)$, and the proof is finished.

Since Hilbert spaces are $U M D$ spaces, we obtain as immediate consequence the following corollary.

Corollary 3.5. Let $H$ be a Hilbert space and let $T \in \mathcal{B}(H)$ be an analytic operator; assume that (2.10) is fulfilled. Then, the following assertions are equivalent.

(i) Equation (2.2) is well-posed.

(ii) $\sup _{z \in \mathbb{T}_{r}^{\alpha}, z \neq \alpha r}\left\|(z-r)^{2}\left((z-r)^{2}-r^{2}(I-T)\right)^{-1}\right\|<\infty$

(iii) Equation (2.2) has discrete maximal regularity.

Remark 3.6. a) We note that when (2.2) has discrete maximal regularity, then $\left(x_{n}\right) \in$ $l_{p, r}^{2}\left(\mathbb{Z}_{+} ; X\right) \cap l_{p, I-T}\left(\mathbb{Z}_{+} ; X\right)$ whenever $\left(f_{n}\right) \in l_{p}\left(\mathbb{Z}_{+} ; X\right)$. It establishes an isomorphism between the set of data $\left(f_{n}\right)$ and the set of solutions $\left(x_{n}\right)$. 
b) For each $f=\left(f_{n}\right) \in l_{p}\left(\mathbb{Z}_{+} ; X\right)$ and under the equivalent conditions of the above theorem, there exists a constant $C>0$ such that $\left\|\Delta_{r}^{2} x\right\|_{p}+\left\|r^{2}(I-T) x\right\|_{p} \leq C\|f\|_{p}$, for all $x=\left(x_{n}\right) \in l_{p, r}^{2}\left(\mathbb{Z}_{+} ; X\right) \cap l_{p, I-T}\left(\mathbb{Z}_{+} ; X\right)$, that is, the application $R: l_{p}\left(\mathbb{Z}_{+} ; X\right) \rightarrow$ $l_{p, r}^{2}\left(\mathbb{Z}_{+} ; X\right) \cap l_{p, I-T}\left(\mathbb{Z}_{+} ; X\right)$ given by $\left(f_{n}\right) \mapsto\left(x_{n}\right)$ is continuous.

Let $f=\left(f_{n}\right) \in l_{p}\left(\mathbb{Z}_{+} ; X\right)$. We finish this paper with an application to the following semilinear evolution problem

$$
\Delta_{r}^{2} x-r^{2}(I-T) x=G(x)+\rho f,
$$

where $G$ is a Frechét differentiable function and $\rho>0$ is a small parameter.

Theorem 3.7. Let $X$ be a $U M D$ space and $T \in \mathcal{B}(X)$ be an analytic operator. Assume that (2.10) is fulfilled and assume that

(i) the set $\{M(z):|z|=\alpha r, z \neq \alpha r\}$ is R-bounded.

(ii) $G$ maps $l_{p, r}^{2}\left(\mathbb{Z}_{+} ; X\right) \cap l_{p, I-T}\left(\mathbb{Z}_{+} ; X\right)$ into $l_{p}\left(\mathbb{Z}_{+} ; X\right) ; G(0)=0 ; G$ is a continuously (Frechét) differentiable function at $x=0$ and $G^{\prime}(0)=0$.

Then, there exists $\rho^{*}>0$ such that the equation (3.2) is solvable for each $\rho \in\left[0, \rho^{*}\right)$, with solution $x_{\rho}:=\left(x_{n}\right) \in l_{p, r}^{2}\left(\mathbb{Z}_{+} ; X\right) \cap l_{p, I-T}\left(\mathbb{Z}_{+} ; X\right)$.

Proof. Define the operator $L: l_{p, r}^{2}\left(\mathbb{Z}_{+} ; X\right) \cap l_{p, I-T}\left(\mathbb{Z}_{+} ; X\right) \rightarrow l_{p}\left(\mathbb{Z}_{+} ; X\right)$ by $L(x)=$ $\Delta_{r}^{2} x-r^{2}(I-T) x$. We consider for $\rho \in(0,1)$ the one parameters family

$$
H[x, \rho]:=-L(x)+G(x)+\rho f .
$$

Keeping in mind that $G(0)=0$ we see that $H[0,0]=0$. Also, by hypothesis, $H$ is continuously differentiable at $(0,0)$. We observe that $L$ is an isomorphism onto. In fact, by uniqueness $L$ is injective. By Theorem 3.4, $L$ is surjective. By definition of the norm in (3.1), $L$ is bounded. Now, the claim follows by the Open Mapping Theorem. Hence the partial Frechét derivative $H_{(0,0)}^{1}=-L$ is invertible. The conclusion of the theorem now follows from the Implicit Function Theorem (see [27, Theorem 17.6]).

In the very special case that $T=I$ and $X=\mathbb{R}$ we obtain the following scalar result which, in our best know, is also new.

Corollary 3.8. Assume that (2.10) is fulfilled, let $f=\left(f_{n}\right)$ be in $l_{p}\left(\mathbb{Z}_{+} ; \mathbb{R}\right)$. Assume that $G$ is like in the preceding theorem. Then, there exists $\rho^{*}>0$ such that the discrete time evolution equation

$$
\Delta_{r}^{2} x=G(x)+\rho f
$$

is solvable for each $\rho \in\left[0, \rho^{*}\right)$, with solution $x_{\rho}:=\left(x_{n}\right) \in l_{p, r}^{2}\left(\mathbb{Z}_{+} ; \mathbb{R}\right) \cap l_{p}\left(\mathbb{Z}_{+} ; \mathbb{R}\right)$.

Next, we exhibit a concrete example of mapping $G$ like in Theorem 3.7.

Example 3.9. Let $H$ be a Hilbert space and $T \in \mathcal{B}(H)$. Let $G$ be a map from $l_{p, r}^{2}\left(\mathbb{Z}_{+} ; H\right) \cap$ $l_{p, I-T}\left(\mathbb{Z}_{+} ; H\right)$ into $l_{p}\left(\mathbb{Z}_{+} ; H\right)$ defined by

$$
G(h)(n)=<\Delta_{r}^{2} h_{n}, \Delta_{r}^{2} h_{n}>.
$$

We observe that

$$
\|G(h)\|_{p} \leq\left\|\Delta_{r}^{2} h\right\|_{\infty}\left\|\Delta_{r}^{2} h\right\|_{p}, h \in l_{p, r}^{2}\left(\mathbb{Z}_{+} ; X\right) \cap l_{p, I-T}\left(\mathbb{Z}_{+} ; X\right),
$$


whence $G$ is well defined and $G(0)=0$. Also, we get

$$
G^{\prime}(x)(h)=2<\Delta_{r}^{2} x, \Delta_{r}^{2} h>, x, h \in l_{p, r}^{2}\left(\mathbb{Z}_{+} ; X\right) \cap l_{p, I-T}\left(\mathbb{Z}_{+} ; X\right),
$$

so $G^{\prime}(0)=0$. On the other hand, we can infer that

$$
\left\|G^{\prime}(x)-G^{\prime}\left(x^{0}\right)\right\| \leq 2\left\||| x-x^{0} \mid\right\|, x, x^{0} \in l_{p, r}^{2}\left(\mathbb{Z}_{+} ; X\right) \cap l_{p, I-T}\left(\mathbb{Z}_{+} ; X\right),
$$

then $G$ is a continuously (Frechét) differentiable function.

Example 3.10. Assume that (2.10) is fulfilled; let $\mu$ be a positive real number and $f=$ $\left(f_{n}\right) \in l_{p}\left(\mathbb{Z}_{+} ; \mathbb{R}\right)$. Then, there exists $\rho^{*}>0$ such that the discrete time evolution problem

$$
\left\{\begin{array}{l}
\Delta_{r}^{2} x_{n}=\left[\Delta_{r}^{2} x_{n}\right]^{\mu}+\rho f_{n}, n \in \mathbb{Z}_{+}, \\
x_{0}=x_{1}=0 .
\end{array}\right.
$$

is solvable for each $\rho \in\left[0, \rho^{*}\right)$, with solution $x_{\rho}:=\left(x_{n}\right) \in l_{p, r}^{2}\left(\mathbb{Z}_{+} ; \mathbb{R}\right) \cap l_{p}\left(\mathbb{Z}_{+} ; \mathbb{R}\right)$.

\section{REFERENCES}

[1] R.P. Agarwal, Difference Equations and Inequalities, Monographs and Textbooks in Pure and Applied Mathematics, 228, Marcel Dekker, New York, 2000.

[2] H. Amann, Quasilinear parabolic functional evolution equations. In: M. Chipot, H. Ninomiya (editors), Recent Advances in Elliptic and Parabolic Issues. Proc. of the 2004 Swiss - Japanese Seminar, World Scientific, (2006), 19 - 44.

[3] H. Amann. Linear and Quasilinear Parabolic Problems. Monographs in Mathematics. 89. Basel: Birkhäuser Verlag, 1995.

[4] W. Arendt, S. Bu, The operator-valued Marcinkiewicz multiplier theorem and maximal regularity, Math. Z. 240 (2002), 311-343.

[5] W. Arendt, S. Bu, Operator-valued Fourier multiplier on periodic Besov spaces and applications, Proc. Edim. Math. Soc., 47 (2) (2004), 15-33.

[6] W. Arendt, Semigroups and evolution equations: functional calculus, regularity and kernel estimates, Evolutionary equations. Vol. I, 1-85, Handb. Differ. Equ., North-Holland, Amsterdam, 2004.

[7] A. Ashyralyev, C. Cuevas and S.Piskarev, On well-posedness of difference schemes for abstract elliptic problems in $L^{p}([0, T] ; E)$ spaces, Numer. Funct. Anal. Optim. 29 (1-2), (2008), 43-65.

[8] A. Ashyralyev, S. Piskarev, L. Weis, On well-posedness of difference schemes for abstract parabolic equations in $L^{p}([0, T] ; E)$ spaces, Numer. Funct. Anal. Optim. 23 (2002), no. 7-8, 669-693.

[9] A.Drozdowicz, J. Popenda, Asymptotic behavior of the solutions of the second order difference equation. Proc. Amer. Math. Soc. 99(1), (1987), 135-140.

[10] A. Bátkai, E. Fasanga, R. Shvidkoy, Hyperbolicity of delay equations via Fourier multiplier, Acta Sci. Math. (Szeged) 69 (2003), 131-145.

[11] E. Berkson, T.A. Gillespie. Spectral decompositions and harmonic analysis on UMD-spaces, Studia Math. 112 (1994), 13-49.

[12] S. Blunck, Maximal regularity of discrete and continuous time evolution equations, Studia Math. 146(2) (2001), 157-176.

[13] S. Blunck, Analyticity and discrete maximal regularity of $L_{p}$-spaces, J. Funct. Anal., 183 (1) (2001), 211-230.

[14] J. Bourgain, Some remarks on Banach spaces in which martingale differences sequences are unconditional, Arkiv Math., 21 (1983), 163-168.

[15] D.L. Burkholder, A geometrical condition that implies the existence of certain singular integrals on Banach-space-valued functions. In: Conference on Harmonic Analysis in Honour of Antoni Zygmund, 
Chicago 1981, W. Becker, A.P. Calderón, R. Fefferman, P.W. Jones (eds), Belmont, Cal. Wadsworth (1983), 270-286.

[16] Ph. Clément, G. Da Prato, Existence and regularity results for an integral equation with infinite delay in a Banach space, Integral Equations Operator Theory, 11 (1988), 480-500.

[17] Ph. Clément, B. de Pagter, F.A. Sukochev, M. Witvliet, Schauder decomposition and multiplier theorems, Studia Math., 138 (2000), 135-163.

[18] Ph. Clément, S.O. Londen, and G. Simonett, Quasilinear evolutionary equations and continuous interpolation spaces, J. Differential Equations, 196 (2) (2004), 418-447.

[19] C. Cuevas, C. Lizama, Maximal regularity of discrete second order Cauchy problems in Banach spaces, J. Differ. Equ. Appl., 13 (12) (2007), 1129-1138.

[20] C. Cuevas, C. Vidal, A note on discrete maximal regularity for functional difference equations with infinite delay. Adv. Difference Equ.( 2006), 1-11.

[21] C. Cuevas, C. Lizama, Semilinear evolution equations on discrete time and maximal regularity, submitted.

[22] C. Cuevas, C. Lizama, Semilinear evolution equations of second order via maximal regularity, Adv. Difference Equ., (2008), doi:10.1155/2008/316207.

[23] R. Denk, M. Hieber and J. Prüss, R-boundedness, Fourier multipliers and problems of elliptic and parabolic type, Mem. Amer. Math. Soc., 166 (788), 2003.

[24] N. Dungey, A note on time regularity for discrete time heat kernels, Semigroup Forum, 72 (2006), 404-410.

[25] S. Elaydi, An introduction to difference equations, Undergraduate Texts in Mathematics, 3rd. ed. Springer Verlag, 2005.

[26] M. Geissert, Maximal $L^{p}$ regularity for parabolic difference equations, Math. Nach., (16) 279 (2006), $1787-1796$.

[27] D. Gilbarg, N.S. Trudinger, Elliptic Partial Differential Equations of Second Order, Grundlehren der mathematischen Wissenschaften 224, Springer-Verlag, 1983.

[28] M. Girardi, L. Weis, Operator-valued Fourier multiplier theorems on Besov spaces, Math. Nachr., 251 (2003), 34-51.

[29] D. Guidetti and S. Piskarev, Stability of the Crank-Nicolson scheme and maximal regularity for parabolic equations in $C^{\theta}(\bar{\Omega})$ spaces, Numer. Funct. Anal. Optim., 20 (3-4) (1999), 251-277.

[30] N.J. Kalton, P. Portal, Remarks on $l^{1}$ and $l^{\infty}$ maximal regularity for power bounded operators, Preprint 2007.

[31] V. Keyantuo, C. Lizama, Maximal regularity for a class of integro-differential equations with infinite delay in Banach spaces, Studia Math., 168 (1) (2005), 25-49.

[32] V. Keyantuo, C. Lizama, Fourier multipliers and integro-differential equations in Banach spaces, J. London Math. Soc., 69 (3) (2004), 737-750.

[33] V. Keyantuo, C. Lizama, Periodic solutions of second order differential equations in Banach spaces, Math. Z., 253 (3) (2006), 489-514.

[34] V. Keyantuo, C. Lizama, Hölder continuous solutions for integro-differential equations and maximal regularity. J. Differential Equations, 230 (2006), 634-660.

[35] V.B. Kolmanovskii, E. Castellanos-Velasco and J.A. Torres-Munoz, A survey: stability and boundedness of Volterra difference equations, Nonlinear Anal., 53 (7) (2003), 861-928.

[36] Y. Latushkin, F. Räbiger, Operator valued Fourier multiplier and stability of strongly continuos semigroup, Integral Equations Operator Theory 51 (3) (2005), 375-394.

[37] P. Portal, Discrete time analytic semigroups and the geometry of Banach spaces, Semigroup Forum, 67 (2003), 125-144.

[38] P. Portal, Maximal regularity of evolution equations on discrete time scales, J. Math. Anal. Appl., 304 (2005), 1-12.

[39] L. Weis, Operator-valued Fourier multiplier theorems and maximal $L_{p}$-regularity, Math. Ann., 319 (2001), 735-758

[40] L. Weis, A new approach to maximal $L_{p}$-regularity, Lecture Notes Pure Appl. Math., 215, Marcel Dekker, New York, 2001, 195-214.

[41] F. Zimmermann. On vector-valued Fourier multiplier theorems. Studia Math. 93 (1989), 201-222. 
Universidade Federal de Pernambuco, Departamento de Matemática, Av. Prof. Luiz Freire, S/N, Recife-PE, CEP. 50540-740, Brazil.

E-mail address: (Airton Castro)airton@dmat.ufpe.br

E-mail address: (Claudio Cuevas)cch@dmat.ufpe.br

Universidad de Santiago de Chile, Departamento de Matemática, Facultad de Ciencias, Casilla 307-Correo 2, Santiago-Chile.

E-mail address: (Carlos Lizama)clizama@usach.cl 